there was no additional pain or distress for the animal. The issue of cost drives many improvements to processes. Rather than unofficially reprimanding these two researchers, they should be encouraged to continue creative thinking. They were right to assume that the IACUC would be happy that they're using fewer animals.

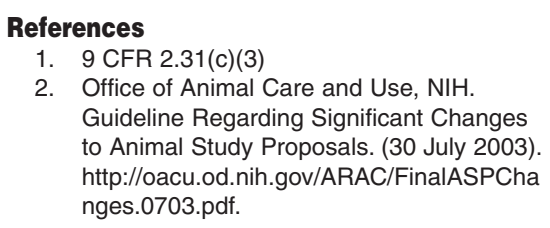

2. Office of Animal Care and Use, $\mathrm{NIH}$. Guideline Regarding Significant Changes to Animal Study Proposals. (30 July 2003). http://oacu.od.nih.gov/ARAC/FinalASPCha nges.0703.pdf.

Kuhlman is the Head of Veterinary Surgical Services at Pfizer Inc., Kalamazoo, MI.

\section{A Pig in Time Saves Nine}

\section{Lori R. Hill, DVM, DACLAM and Torsten Hopp PhD}

It seems that the researchers in this scenario were well meaning in that they were, in fact, practicing the 'reduction' component of the 3Rs by making more extensive use of one animal rather than using two. Doing multiple terminal procedures on a single animal can maximize the use of animals as a means of reduction ${ }^{1}$. In addition, both of the researchers reached their objectives without causing further pain or distress to the animal. According to the scenario, it seems that Bertucci and Spinoza did not knowingly commit a protocol violation. In fact, all procedures were already approved, just not under one protocol umbrella. In our opinion, however, the objective of cost reduction alone is not a valid justification.

There is a fairly straightforward way that this problem could have been avoided. The researchers could have added each other and any other manipulators to one another's protocols. In addition, they could have briefly described their use of one animal for both protocols and each referenced the other protocol. The USDA category would not change because both were doing terminal procedures. The IACUC would probably not have considered these types of changes to be substantive and might have approved them administratively, in a timely manner ${ }^{1-3}$.

There is still the question of under which of the two protocols the animals would be purchased, housed and manipulated, and how they should be counted. One should count the animals only once, regardless of the number of proposals in which they were used ${ }^{1,4}$. If both protocols were amended to reference all potential procedures and manipulators, we would have suggested that the researchers alternate in using their respective protocols. This situation obviously does not allow the transfer of animals from one protocol to the other.

We are inclined to suggest a solution similar to that of Lockridge by requiring that both researchers amend their protocols to cover the combination of procedures and manipulators. Because the animal was not subjected to further pain and distress, and all procedures were approved (albeit under separate protocols), that amendment of the protocols would be sufficient. The USDA, APHIS Animal Care Policy \#17, specifically discusses the use of animals on more than one protocol ${ }^{4}$.

The issue of whether this situation is a 'federal case' (i.e., reportable) seems clearcut. The PHS Policy states that the IACUC, through the IO, must report to OLAW "serious or continuing non-compliance with [PHS] Policy, any serious deviation from the provisions of the Guide, or any suspension of an activity by the IACUC 5 ." The Animal Welfare Regulations only require reporting to APHIS when an activity is suspended ${ }^{6}$. The scenario described in this column does not fulfill any of the requirements for reporting.

The Great Eastern IACUC needs to develop a policy for the use of animals on multiple protocols. The existence of such a policy to educate investigators could have avoided the scenario described here .

\footnotetext{
References

1. Silverman, J., Suckow, M.A. \& Murthy, S., eds. The IACUC Handbook (CRC Press,
}

Boca Raton, FL, 2000).

2. Wolff, A., Garnett, N., Potkay, S., Wigglesworth, C., Doyle, D. \& Thornton, V. Frequently asked questions about the Public Health Service Policy on Humane Care and Use of Laboratory Animals. Lab Anim. (NY) 24(9), 33-36 (2003).

3. National Institutes of Health. Office of Extramural Research. Revised Guidance Regarding IACUC Approval of Changes in Personnel Involved in Animal Activities. Notice: NOT-OD-03-046 (6 June 2003).

4. USDA APHIS. Animal Care Policy \#17, Annual Report for Research Facilities (17 March 1999).

5. Public Health Service. Public Health Service Policy on Humane Care and Use of Laboratory Animal IV.F.3 (US Department of Health and Human Services, Washington, DC, 1986).

6. 9 CFR, Part 2, Subchapter A, Regulations.

Hill is Institutional Veterinarian and Hopp is IACUC Administrator, Baylor College of Medicine, Houston, TX.

\section{Don't Cross That Line!}

Markus Josten, Dr. med. vet.

To make their lives a little bit easier, two scientists have changed their protocols. "Easy" in this context means "cheaper", because they agreed on using one pig where, according to the protocols, they should have used two. From the 3R's standpoint, they have reached the very desirable goal of using the smallest number of animals possible in animal experimental work. From their point of view it is an issue of nonsurvival surgery, and one could even have some sympathy for their idea of pig sharing, with both of them using different organs of a single animal that will not recover from this procedure anyway. However, there are certain rules in our world, and they exist not solely to make our lives more difficult or complicated, as they sometimes seem to do. It is the initial intention of these rules to provide a clear framework in which we can live our lives. It is obligatory for everyone to stay inside these boundaries, because the freedom of the one comes to an end where the freedom of the other is affected. As a matter of fact, we might sometimes come close to the 